\title{
ARTICLE OPEN \\ LncRNAs LCETRL3 and LCETRL4 at chromosome 4q12 diminish EGFR-TKIs efficiency in NSCLC through stabilizing TDP43 and EIF2S1
}

\author{
Yankang $\mathrm{Li}^{1}$, Yue Shen ${ }^{1}$, Mengyu Xie ${ }^{1}$, Bowen Wang ${ }^{1}$, Teng Wang ${ }^{1}$, Jiajia Zeng ${ }^{2}$, Hui Hua ${ }^{2}$, Jinming Yu ${ }^{3,4 \bowtie}$ and Ming Yang $\mathbb{D}^{1,2,3 凶}$
}

Epidermal growth factor receptor (EGFR)-tyrosine kinase inhibitors (TKIs) are effective targeted therapy drugs for advanced nonsmall cell lung cancer (NSCLC) patients carrying sensitized EGFR mutations. The rapid development of EGFR-TKIs resistance represents a major clinical challenge for managing NSCLC. The chromosome $4 q 12$ is the first genome-wide association study (GWAS)-reported locus associated with progression-free survival (PFS) of NSCLC patients treated with EGFR-TKIs. However, the biological significance of the noncoding transcripts at $4 q 12$ in NSCLC remains elusive. In the present study, we identified two $4 q 12$ long noncoding RNAs (IncRNAs) LCETRL3 and LCETRL4 which could significantly dimmish EGFR-TKIs efficiency. In line with their oncogenic role, evidently higher LCETRL3 and LCETRL4 levels were observed in NSCLC tissues as compared with normal specimens. Importantly, IncRNA LCETRL3 can interact with oncoprotein TDP43 and inhibit ubiquitination and degradation of TDP43. Similarly, InCRNA LCETRL4 can bind and stabilize oncoprotein EIF2S1 through reducing ubiquitin-proteasome degradation of EIF2S1. In particular, elevated levels of LCETRL3 or LCETRL4 in NSCLC cells resulted in stabilization of TDP43 or EIF2S1, increased levels of NOTCH1 or phosphorylated PDK1, activated AKT signaling and, thus, EGFR-TKIs resistance. Taken together, our data revealed a novel model that integrates two IncRNAs transcribed from the $4 q 12$ locus into the regulation of EGFR-TKIs resistance in NSCLC. These findings shed new light on the importance of functionally annotating IncRNAs in the GWAS loci and provided insights to declare novel druggable targets, i.e., IncRNAs, which may unlock the therapeutic potential of EGFR-TKIs resistant NSCLC in the clinic.

\section{INTRODUCTION}

Lung cancer is one of the most common malignancies in the world. ${ }^{1}$ Non-small cell lung cancer (NSCLC) accounts for about $85 \%$ of all incident lung cancer. The prognosis of NSCLC is still poor, with the 5-year survival rate of $15 \% .^{1}$ In clinics, the epidermal growth factor receptor (EGFR)-tyrosine kinase inhibitors (TKIs) have been proven to be effective for the treatment of advanced NSCLC patients carrying sensitized EGFR mutations including the exon 19 deletion or the L858R mutation., ${ }^{2,3}$ Gefitinib is the firstly approved orally bioavailable, competitive, reversible EGFR-TKI for NSCLC with EGFR mutations. The large randomized phase III IPASS trail indicated a beneficial effect on progression-free survival (PFS) in the NSCLC patients with EGFR mutation treated with gefitinib compared to patients treated with chemotherapy. ${ }^{4,5}$ Suppression of EGFR via Gefitinib impacts multiple signaling pathways involved in tumor proliferation such as the PI3K/AKT pathway. ${ }^{4-6}$ Despite the promising anti-NSCLC effects, almost all patients underwent EGFR-TKIs resistance sooner or later. Importantly, short PFS was observed in a large portion of advanced NSCLC patients after EGFR-TKIs therapy. ${ }^{2,3}$ Therefore, it is essential to declare biological mechanisms responsible for the development of EGFR-TKIs resistance in NSCLC.
Germline genetic variations, i.e., single-nucleotide polymorphisms (SNPs), could impact the pharmacokinetic and/or pharmacodynamic profiles of EGFR-TKIs in NSCLC patients. ${ }^{7-12}$ Interestingly, genome-wide association studies (GWASs) have identified the chromosome $4 q 12$ locus significantly associated with PFS of advanced NSCLC patients treated with EGFR-TKIs in several independent cohorts. ${ }^{7}$ It has also been reported that CLOCK, $N M U$ and SRD5A3 in the $4 q 12$ locus might be candidate proteincoding genes involved in NSCLC prognosis. ${ }^{7}$ However, it is still largely unclear how the $4 q 12$ noncoding genes underly the biology of EGFR-TKIs resistance of NSCLC.

Long noncoding RNAs (IncRNAs) are a group of ncRNAs with a length greater than 200nt, which play a vital part in human cancers including NSCLC. ${ }^{13-15}$ Accumulating evidences elucidated that multiple IncRNAs contributed to the development of EGFRTKIs resistance in NSCLC. ${ }^{16-21}$ For instance, IncRNA H19 conferred to EGFR-TKIs resistance via interacting and upregulating PKM2 and increasing levels of AKT phosphorylation in NSCLC. ${ }^{18}$ However, the role of IncRNAs transcribed from the $4 q 12$ locus in NSCLC development and progression remains to be explored.

In the current study, we systematically examined five candidate IncRNAs at $4 q 12$, which are named as Lung Cancer EGFR-TKIs

\footnotetext{
${ }^{1}$ Cheeloo College of Medicine, Shandong University, Jinan, Shandong Province 250112, China; ${ }^{2}$ Shandong Provincial Key Laboratory of Radiation Oncology, Cancer Research

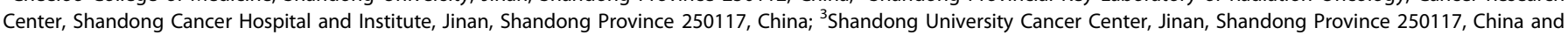

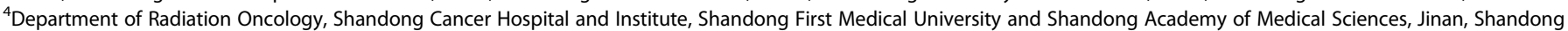
Province 250117, China

Correspondence: Jinming Yu (sdyujinming@126.com) or Ming Yang (aaryoung@yeah.net)
}

Received: 28 June 2021 Revised: 13 October 2021 Accepted: 1 December 2021

Published online: 31 January 2022 
Resistance Long noncoding RNA 1, 2, 3, 4, and 5 (LCETRL1, LCETRL2, LCETRL3, LCETRL4, and LCETRL5) (Supplementary Table 1). We identified IncRNAs LCETRL3 and LCETRL4 functioning as the novel pharmacogenomic regulators of EGFR-TKls resistance. In NSCLC cells, high levels of LCETRL3 or LCETRL4 could evidently promote malignant proliferation and diminish EGFR-TKIs efficiency in vitro and in vivo. In support of this, IncRNAs LCETRL3 and LCETRL4 are highly expressed in NSCLC specimens. Importantly, LCETRL3 or LCETRL4 could stabilize oncoprotein TDP43 or EIF2S1, upregulate NOTCH1 expression or phosphorylated PDK1 levels, and, thus, lead to the activated AKT signaling of NSCLC cells.

\section{RESULTS}

Elevated expression of IncRNAs LCETRL3 and LCETRL4 at chromosome $4 q 12$ in NSCLC tissues

To reveal whether IncRNAs transcribed from the chromosome $4 q 12$ locus are involved in NSCLC development, we firstly examined expression levels of five candidate IncRNAs (LCETRL1, LCETRL2, LCETRL3, LCETRL4, and LCETRL5) (Fig. 1a and Supplementary Table 1 ) in paired NSCLC and normal tissues of Discovery cohort $(n=20)$. We found that IncRNAs LCETRL3 and LCETRL4 showed significantly elevated expression in cancerous tissues compared to normal lung specimens (both $P<0.05$ ) (Fig. 1b). Consistently, we found evidently increased levels of LCETRL3 and LCETRL4 in NSCLC specimens of Validation cohort $(n=44)$ (both $P<0.001$ ) (Fig. 1c). We also investigated the aberrant expression of both IncRNAs in the TCGA lung cancer cohort and observed markedly increased LCETRL3 or LCETRL4 expression in NSCLC specimens compared to normal tissues (both $P<0.05$ ) (Fig. 1d). The median follow-up time of all NSCLC patients in both the Discovery cohort and the Validation cohort was thirty months. After the last follow-up, we found that there were a few of NSCLC patients with disease progression. Two patients died and sixteen had disease progression. As a result, we examined the differences of the expression levels of LCETRL3 or LCETRL4 in NSCLC tissues between patients with disease progression and patients without disease progression (Supplementary Fig. 1a and b). We found that there was the significantly elevated expression of LCETRL3 or LCETRL4 in cases with disease progression compared to patients without disease progression (Supplementary Figure $1 \mathrm{a}$ and $1 \mathrm{~b}$ ). These data imply that IncRNAs LCETRL3 and LCETRL4 may act as novel oncogenes during NSCLC progression.

As shown in Fig. 1a, LCETRL3 and LCETRL4 are two adjacent IncRNA genes located in chromosome 4q12. Accumulating evidences indicated that IncRNAs may regulate adjacent gene(s) transcription and expression levels. ${ }^{13-15,22}$ First, we examined whether LCETRL3 and LCETRL4 regulate each other's expression in NSCLC (Supplementary Fig. 1c and d). Silencing of IncRNA

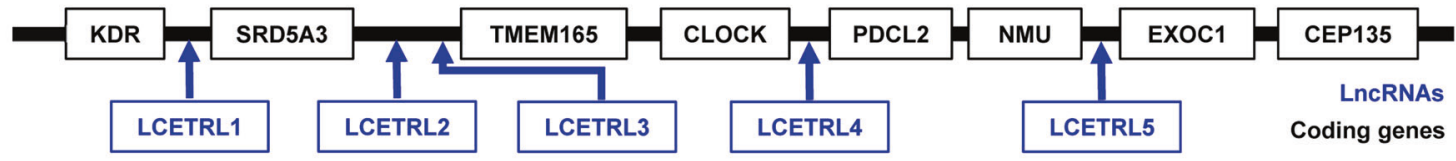

b

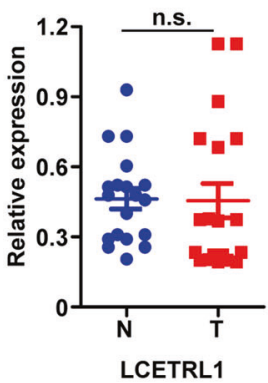

C

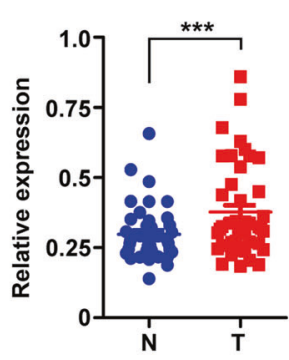

LCETRL3

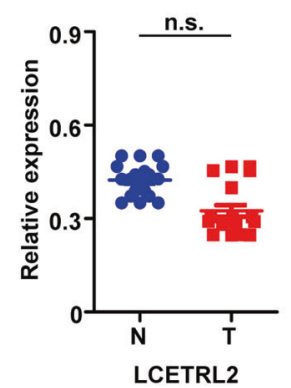

LCETRL2
Discovery cohort $(n=20)$

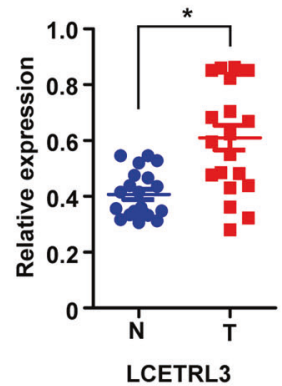

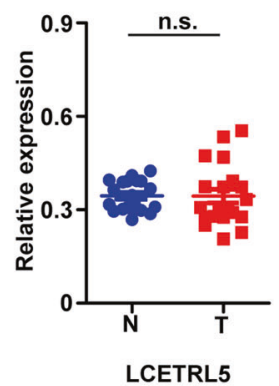

d

TCGA lung cancer cohort

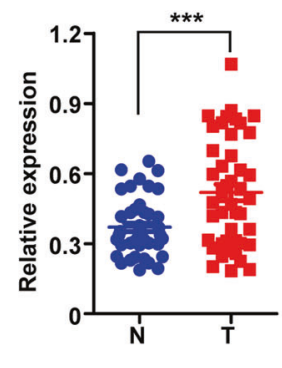

LCETRL4
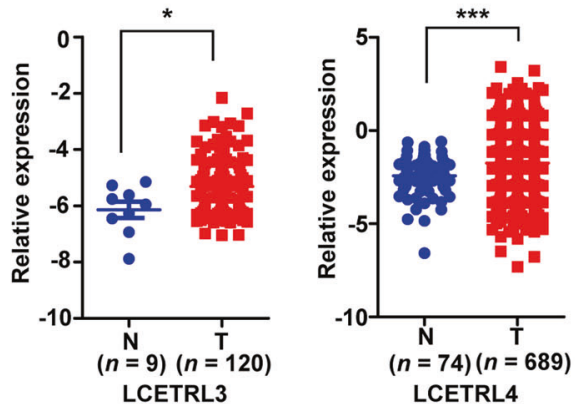

Fig. 1 LCETRL3 and LCETRL4 are two significantly upregulated IncRNAs in NSCLC specimens. (a) Five candidate IncRNAs (LCETRL1, LCETRL2, LCETRL3, LCETRL4 and LCETRL5) locate at 4q12. (b) Relative expression of five candidate IncRNAs in paired NSCLC and normal tissues of Discovery cohort $(n=20)$. (c, d) Significantly elevated expression of IncRNAs LCETRL3 and LCETRL4 in NSCLC specimens of Validation cohort $(n=44)$ and TCGA lung cancer tissues compared to normal tissues. Two-tailed paired $t$ test or two-tailed unpaired $t$ test, n.s., not significant, ${ }^{*} P<0.05,{ }^{* *} P<0.01,{ }^{* * *} P<0.001$ 
LCETRL3 did not impact levels of LCETRL4 in NSCLC cells (Supplementary Fig. 1c). Similarly, siRNAs of LCETRL4 did not influence the expression of LCETRL3 in NSCLC cells (Supplementary Fig. 1c). In support of this notion, no significant expression correlation between LCETRL3 and LCETRL4 was observed in NSCLC tissues of combined samples from the Discovery cohort and Validation cohort $\left(r^{2}=0.024, P=0.238\right)$ (Supplementary Fig. 1d). Therefore, we speculated that LCETRL3 or LCETRL4 may independently play its part during NSCLC development.

LCETRL3 and LCETRL4 promoted malignant proliferation of NSCLC in vitro and in vivo

We further investigated the involvement of IncRNAs LCETRL3 and LCETRL4 in NSCLC in vitro and in vivo. First, multiple NSCLC cell lines were generated via stably silencing LCETRL3 or LCETRL4 by shRNAs or forced-expressing the IncRNAs by plasmids. These NSCLC cells successfully transduced by lentivirus were selected by blasticidin or puromycin. In PC9 and H1299 cells stably expressing LCETRL3 shRNAs, there was significantly decreased expression of the IncRNA (shL3-1 or shL3-2 vs. shNC: $P<0.01$ ) (Fig. 2a). Strikingly over-expressed LCETRL3 was found in PC9 and H1299 cells stalely expressing the LCETRL3 construct (LCETRL3 vs. NC: $P<0.001$ ) (Fig. 2a). As shown in Fig. $2 b$ and $c$, silencing of IncRNA LCETRL3 significantly inhibited proliferation of PC9 and H1299 cells (all $P<0.01$ ); whereas, ectopic LCETRL 3 expression markedly promoted the viability of NSCLC cells (both $P<0.01$ ). Rescue assays indicated that over-expression of LCETRL3 could enhance cell proliferation of NSCLC cells after stable silencing of IncRNA LCETRL3 with shRNAs (Supplementary Fig. 2a). Consistently, knocking-down expression of LCETRL3 suppressed clone formation of PC9 and H1299 cells (Fig. 2d). NSCLC cells with overexpressed IncRNA LCETRL3 showed reinforced clonogenicity (Fig. 2d). We also found that LCETRL3 could profoundly promote the migration and invasion capability of NSCLC cells (Supplementary Figure 3). These data elucidated that IncRNA LCETRL3 acts as a novel oncogene during NSCLC pathogenesis.

There was evidently downregulated expression of IncRNA LCETRL4 in PC9 and H1299 cells stably expressing its shRNAs (shL4-1 or shL4-2 vs. shNC: $P<0.01$ ) (Fig. 2e). On the contrary, markedly over-expression of IncRNA LCETRL4 was observed in both NSCLC cell lines stalely expressing the LCETRL4 construct (LCETRL4 vs. NC: $P<0.001$ ) (Fig. 2e). It has been found that depleted expression of IncRNA LCETRL4 notably inhibited the proliferation of PC9 and H1299 cells (shL4-1 or shL4-2 vs. shNC: $P$ $<0.01$ ) (Fig. 2f). By contrast, the viability of NSCLC PC9 and H1299 cells was significantly promoted by ectopic LCETRL4 expression (LCETRL4 vs. NC: $P<0.01$ ) (Fig. 2g). Moreover, over-expressed LCETRL4 enhance the growth of NSCLC cells after stable knockingdown of IncRNA LCETRL4 (shL4-1 or shL4-2) (Supplementary Fig. 2b). Colony formation assays indicated that the clonogenicity of NSCLC cells was obviously stimulated by IncRNA LCETRL4 (Fig. 2h). We also found that LCETRL4 could remarkably accelerate migration and invasion of PC9 and H1299 cells (Supplementary Fig. 4). Together, these data clarified the oncogenic functions of InCRNA LCETRL4 in NSCLC.

We next assessed the in vivo role of IncRNAs LCETRL3 and LCETRL4 using NSCLC xenografts. Importantly, xenografts stably over-expressing LCETRL3 grew much faster and showed an evident increase in tumor volume and tumor weight compared to the control xenografts $(P<0.001)$ (Fig. $2 \mathrm{i}-\mathrm{k})$. Similarly, growth of the NSCLC xenografts with stably forced expression of IncRNA LCETRL4 was significantly stimulated compared to the control tumors $(P<0.001)$ (Fig. $2 \mathrm{i}-\mathrm{k}$ ). On the contrary, stable silencing of LCETRL3 or LCETRL4 evidently inhibited growth of the NSCLC xenografts compared to the control xenografts (Supplementary Figure $5 \mathrm{a}$ and $5 \mathrm{~b}$ ). Collectively, these results suggested that LCETRL3 and LCETRL4 could promote the malignant proliferation of NSCLC in vivo.
LCETRL3 and LCETRL4 significantly reduce gefitinib sensitivity Considering LCETRL3 and LCETRL4 located at the GWAS-identified $4 q 12$ locus significantly associated with PFS of the EGFR-TKIstreated NSCLC patients, we further evaluated how these IncRNAs impact the gefitinib sensitivity of NSCLC cells. As shown in Fig. 3a, the proliferation of the LCETRL3-KD NSCLC cells (shL3-1 or shL3-2) treated with gefitinib were notably retarded compared to the control cells treated with gefitinib $(P<0.05)$. In line with this, overexpression of LCETRL3 markedly promoted the proliferation of PC9 or $\mathrm{H} 1299$ cells treated with gefitinib $(P<0.05)$ (Fig. $3 b)$. Similarly, silencing of IncRNA LCETRL4 expression notably strengthened the anticancer activities of gefitinib $(P<0.05)$ (Fig. 3c). Ectopic LCETRL4 expression strikingly stimulated cell viability of NSCLC cells treated with gefitinib $(P<0.05)$ (Fig. 3d). We also evaluated the in vivo impacts of IncRNA LCETRL3 or LCETRL4 on gefitinib sensitivity using NSCLC xenografts. We found that gefitinib could obviously suppress the proliferation of NSCLC tumors in mice. However, stably ectopic expression of LCETRL3 or LCETRL4 evidently diminishes gefitinib efficiency in NSCLC xenografts (Fig. 3e-g).

LncRNA LCETRL3 suppressed TDP43 degradation via the ubiquitinproteasome pathway and activated the NOTCH1-PTEN-AKT signaling

To explore how LCETRL3 and LCETRL4 controlling NSCLC development and gefitinib sensitivity, we firstly detected the impacts of both IncRNAs on the expression of adjacent proteincoding genes at 4q12 (Supplementary Figure 6). However, there were no evident expression changes of adjacent protein-coding genes at 4q12 (SRD5A3, KDR, TMEM165, CLOCK, PDCL2, NMU, EXOC1, and CEP135) after silencing of IncRNA LCETRL3 (Supplementary Figure 6a) or LCETRL4 (Supplementary Figure 6b). Considering the crucial functions of IncRNAs as protein-binding scaffolds during carcinogenesis, ${ }^{13-15,23}$ we hypothesized that IncRNAs LCETRL3 and LCETRL4 may bind certain proteins, disturb their downstream signaling and, thus, accelerate the proliferation of NSCLC cells. To test the hypothesis, we firstly examined the cellular localization of IncRNA LCETRL3 in NSCLC cells and found that there was almost equal LCETRL3 in the nucleus or cytoplasm (Fig. 4a). RNA pulldown assays plus mass spectrometry proteomics with the PC9 or $\mathrm{H} 1299$ cell extracts indicated that there were 270 proteins or 84 proteins pulled down by IncRNA LCETRL3 (Fig. 4b). Among these proteins, thirty-seven LCETRL3-pulled down proteins were observed in both NSCLC cell lines (Fig. 4b). We found that TDP43 is the $1^{\text {st }}$ most abundant protein among all proteins identified through mass spectrometry (Supplementary Table 2). Importantly, TDP43 was successfully validated through independent RNA pulldown assays and Western blot in PC9 or H1299 cells (Fig. 4c). Nevertheless, other three candidate proteins (CDCL5, DHX29 and IMPDH2) could not be verified (Fig. 4c). As compared with the IgG control, there was obvious enrichment of IncRNA LCETRL3 in RNA-protein complexes precipitated with the antiTDP43 antibody in both NSCLC cell lines $(P<0.001)$ (Fig. $4 d$ ).

As shown in Fig. $4 \mathrm{e}$, significantly elevated TDP43 expression in cancerous tissues was observed compared to normal tissues in combined samples from the Discovery cohort and Validation cohort $(P<0.01)$, indicating the oncogenic nature of TDP43 in lung cancer. Intriguingly, the knockdown of LCETRL3 markedly downregulated TDP43 protein levels in cells (Fig. 4f). Conversely, overexpressed LCETRL3 increased TDP43 protein expression in cells (Fig. 4f). Rescue assays indicated that over-expression of TDP43 could promote cell viability of NSCLC cells after stable silencing of IncRNA LCETRL3 with shRNAs (Supplementary Fig. 7a). To explore whether IncRNA LCETRL3 may impact TDP43 protein degradation, we detected TDP43 expression in cells treated with the protein synthesis inhibitor CHX. In ectopic LCETRL3 NSCLC cells, the protein levels of TDP43 reduced slower compared to the control cells (Fig. $4 \mathrm{~g}$ ). However, the protein levels of TDP43 reduced much 

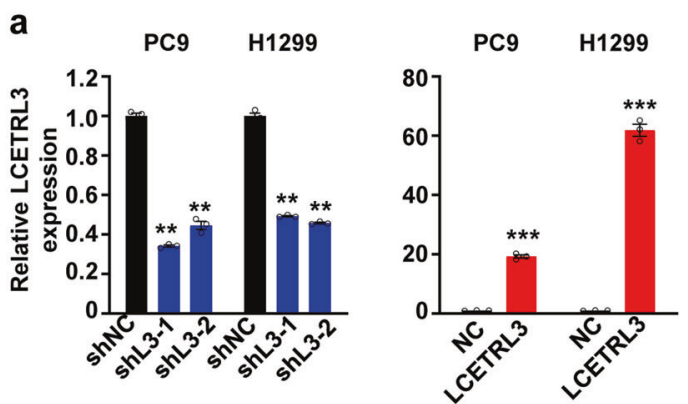

C
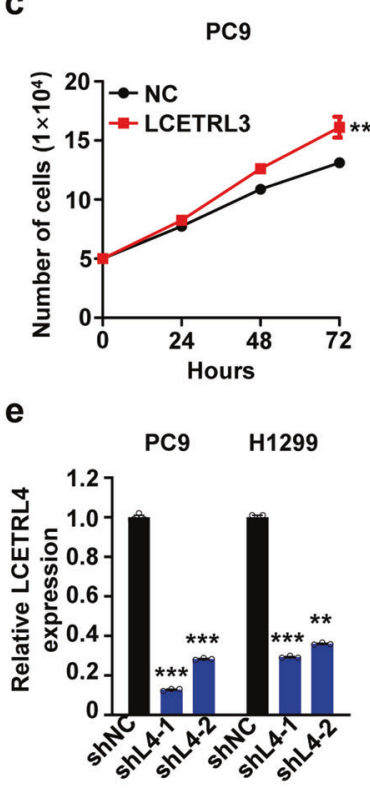

g
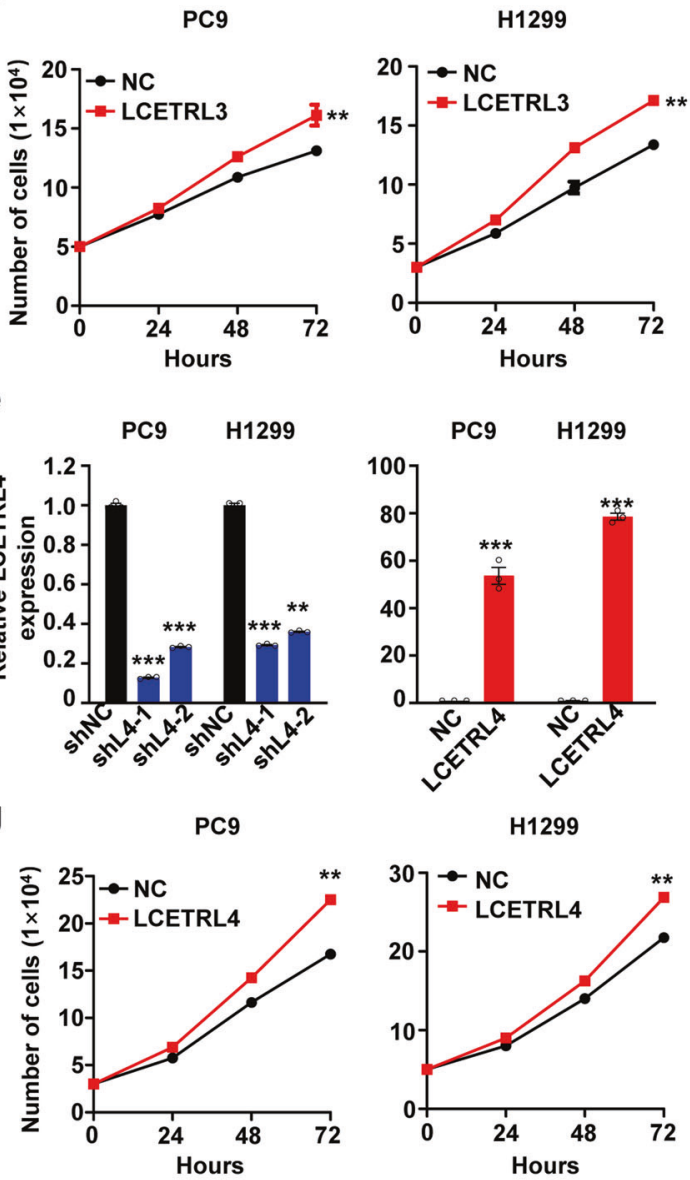

H1299 b

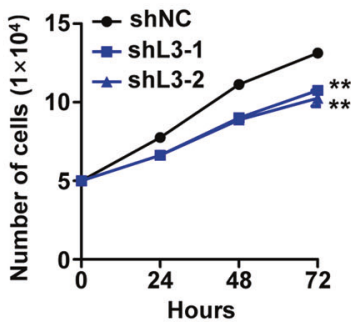

d

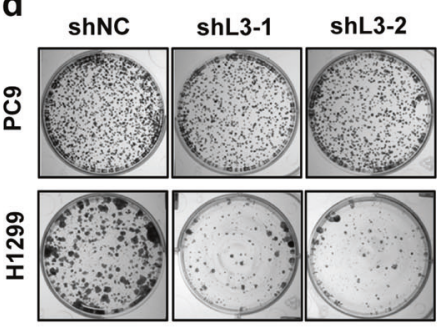

f
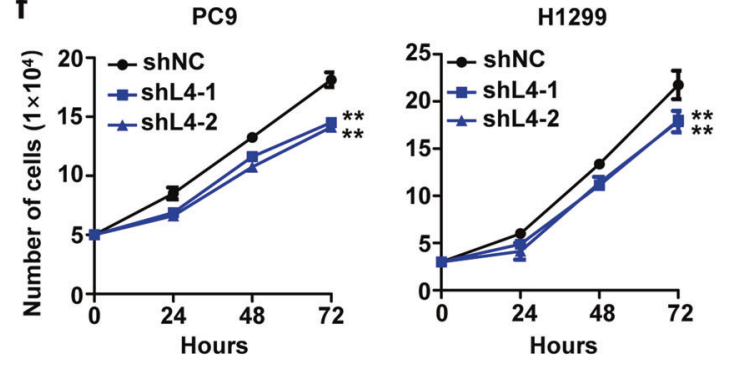

h
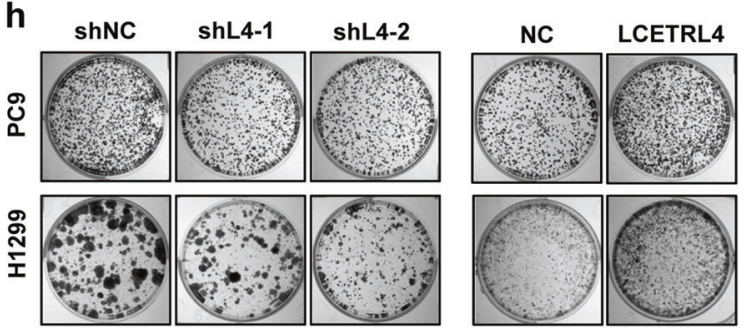

i

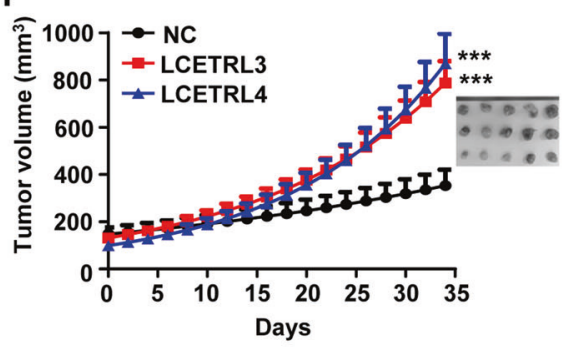

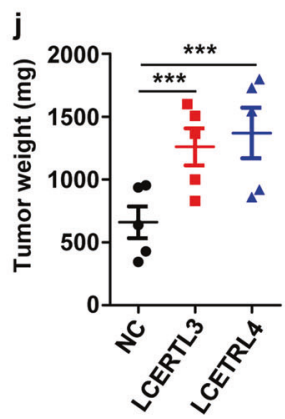

k

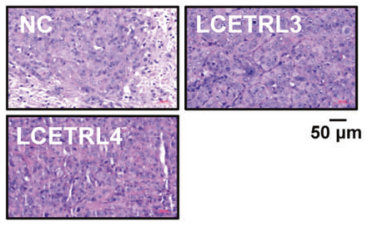

Fig. 2 LncRNAs LCETRL3 and LCETRL4 suppressed malignant proliferation of NSCLC cells in vitro and in vivo. (a-c) Silencing of LCETRL3 with shRNAs inhibited proliferation of PC9 and H1299 cells; whereas overexpressed IncRNA LCETRL3 promoted proliferation of NSCLC cells. (d) LCETRL3 promoted clonogenicity of PC9 and H1299 cells. (e-g) Knocking-down of IncRNA LCETRL4 with shRNAs inhibited proliferation of PC9 and H1299 cells. On the contrary, overexpression of LCETRL4 promoted viability of NSCLC cells. (h) LCETRL4 stimulated colony formation of PC9 and H1299 cells. (i-k) In vivo growth of NSCLC xenografts was promoted by IncRNA LCETRL3 3 or LCETRL4. Two-tailed unpaired $t$ test, ${ }^{* *} P<$ $0.01,{ }^{* * * *} P<0.001$ 


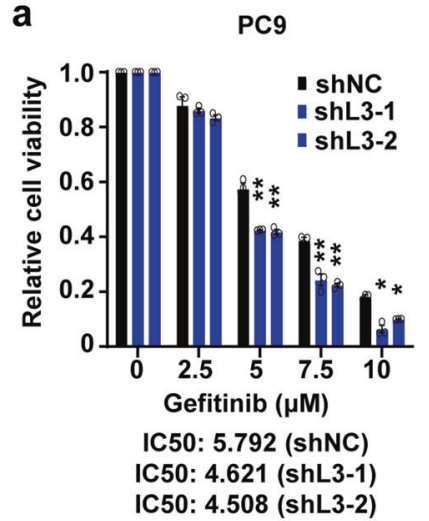

C

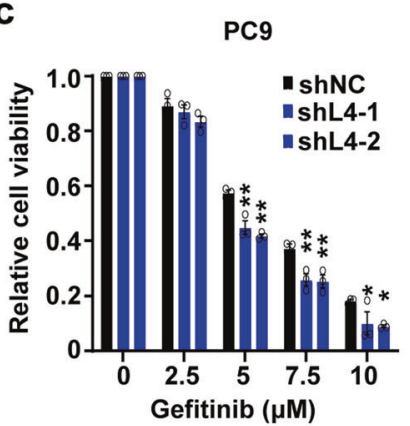

IC50: 5.783 (shNC)

IC50: 4.795 (shL4-1)

IC50: 4.552 (shL4-2)
H1299

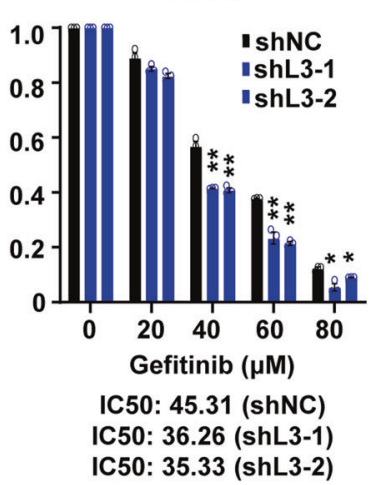

b

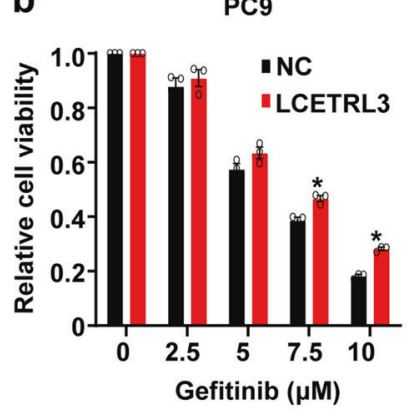

IC50: 5.683 (NC)

IC50: 6.724 (LCETRL3)
H1299

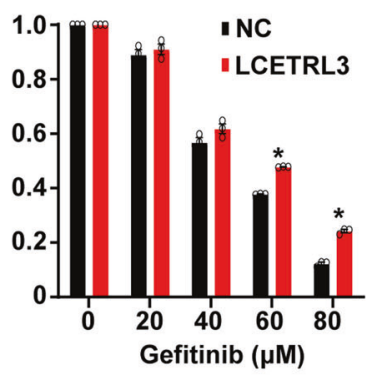

IC50: 46.61 (NC)

IC50: 52.48 (LCETRL3)

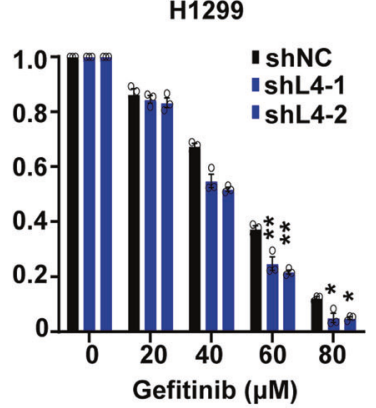

IC50: 48.81 (shNC)

IC50: 40.73 (shL4-1)

IC50: 38.92 (shL4-2) d

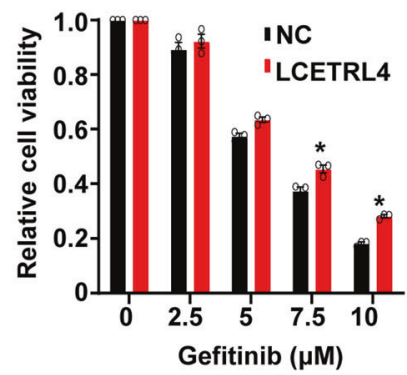

IC50: 5.714 (NC)

IC50: 6.686 (LCETRL4)
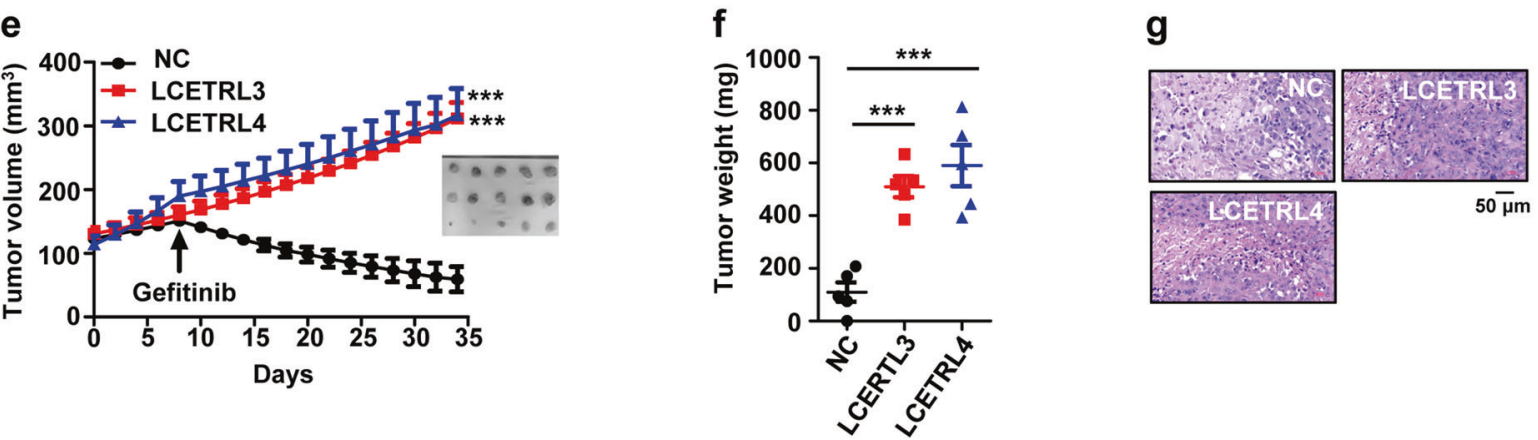

Fig. 3 LncRNAs LCETRL3 and LCETRL4 significantly diminished the antiproliferation effects of gefitinib in vitro and in vivo. (a, b) LncRNA LCETRL3 evidently decreased the antiproliferation effects of gefitinib in PC9 and H1299 cells. (c, d) LncRNA LCETRL4 also obviously diminished the anticancer effects of gefitinib in NSCLC cells. (e-g) LCETRL3 or LCETRL4 significantly reduced efficiency of gefitinib using NSCLC xenografts in vivo. The arrow indicated the start time of gefitinib treatment. Two-tailed unpaired $t$ test, ${ }^{*} P<0.05,{ }^{* *} P<0.01,{ }^{* * * *} P<0.001$

faster in LCETRL3-KD PC9 and H1299 cells compared to the control cells (Fig. 4h), suggesting that LCETRL3 may suppress the proteasome degradation of TDP43 protein. We next investigated if LCETRL3-regulated degradation of TDP43 was mediated by its ubiquitination. After PC9 or H1299 cells were transfected with HAubi, endogenous TDP43 protein was immunoprecipitated. Evidently weakened ubiquitin signals of TDP43 were observed in NSCLC cells with stably ectopic LCETRL3 expression compared with those in the control cells (Fig. 4i). These results elucidated that that IncRNA LCETRL3 promotes TDP43 stabilization via the ubiquitin-proteasome system.

Multiple lines of evidence demonstrated that TDP43 is a DNA/ RNA-binding protein involved in RNA metabolism including the NOTCH1 mRNA. ${ }^{24-26}$ Indeed, silencing of LCETRL3 induced significantly downregulated NOTCH1 expression in both the PC9 and $\mathrm{H} 1299$ cells (shL3-1 and shL3-2 vs. shNC; $P<0.05$ ) (Fig. $4 \mathrm{j}$ and k).
In line with these data, overexpression of LCETRL3 upregulated NOTCH1 expression in NSCLC cells (LCETRL3 vs. NC; $P<0.01$ ) (Fig. $4 \mathrm{j}$ and $\mathrm{k}$ ). NOTCH1 activates AKT through repressing PTEN expression in multiple malignancies including NSCLC. ${ }^{27-29}$ Importantly, knocking-down of LCETRL3 increased PTEN expression and, thus, reduced levels of phosphorylated AKT (T450 and S473) in cells; whereas ectopic LCETRL3 obviously inhibited PTEN expression and enhanced AKT phosphorylation in NSCLC cells (Fig. 4j and $\mathrm{k}$ ). Together, these data indicated that IncRNA LCETRL3 may stabilize TDP43 protein and activate the NOTCH1-PTEN-AKT signaling in NSCLC.

LncRNA LCETRL4 activated the AKT signaling via stabilizing EIF2S1

Subcellular fractionation assays demonstrated that IncRNA LCETRL4 exists nearly in the same amount in the nuclear fraction 
a

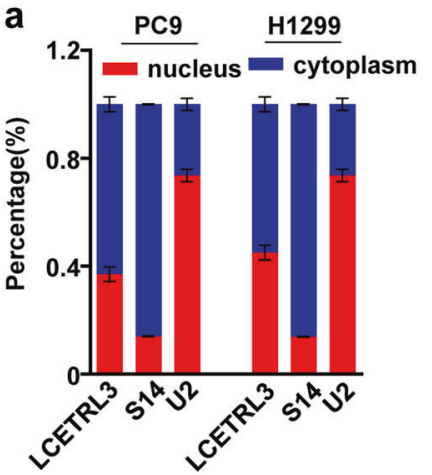

e

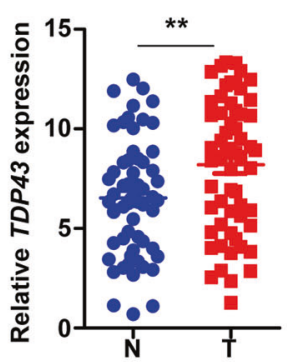

b

Proteins identified via LCETRL3 RNA pulldown

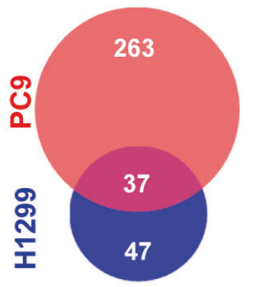

C

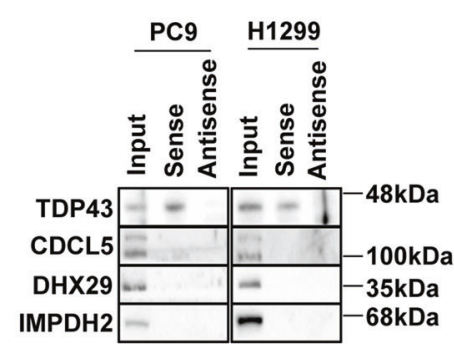

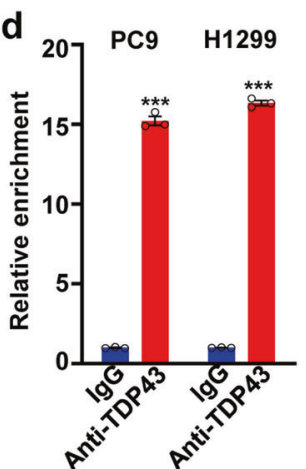

g

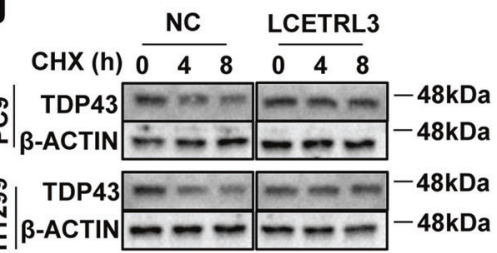

h

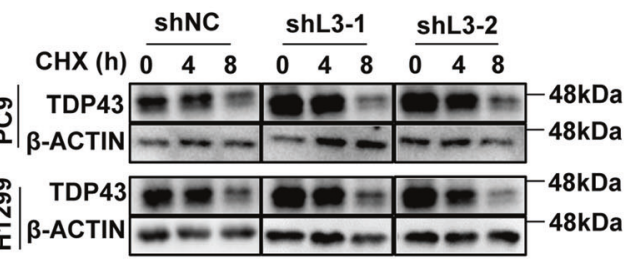

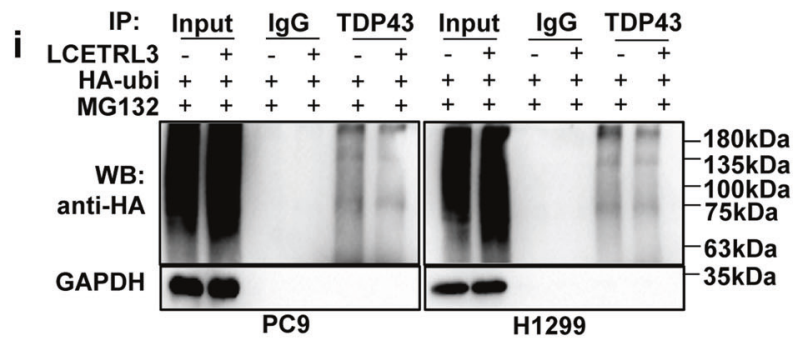

- shNC $=$ shL3-1 $=$ shL3-2 $=$ NC $\square$ LCETRL3
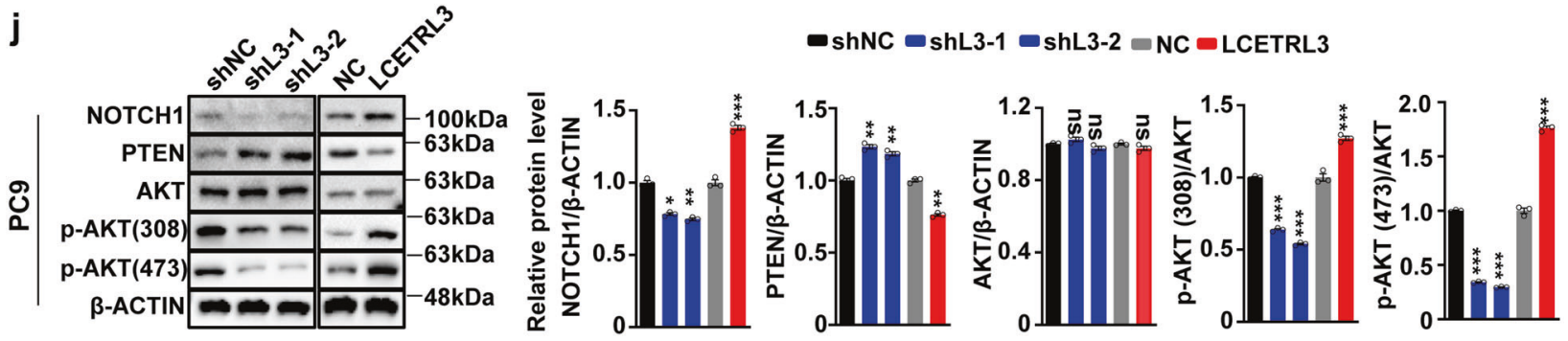

k
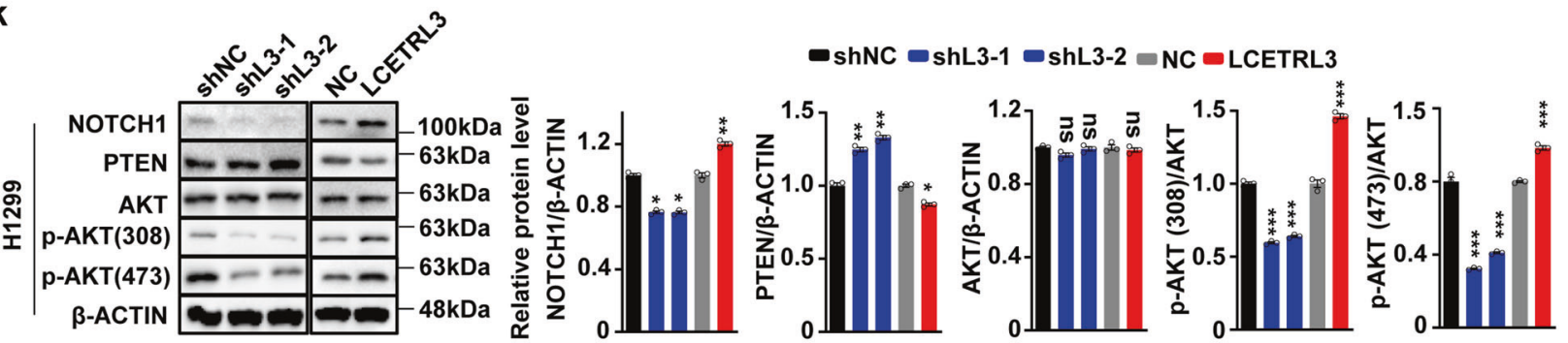

Fig. 4 LnCRNA LCETRL3 interacted with TDP43, prevented TDP43 degradation and activated the AKT signaling. (a) Cellular location of LCETRL3 in NSCLC PC9 and H1299 cells. (b) LCETRL3 pulldown proteins were identified by mass spectrometry in PC9 and H1299 cells. (c) Western blot validation of TDP43 pulled down by IncRNA LCETRL3. (d) LCETRL3 could be precipitated with antibody against TDP43 as compared with the IgG control in PC9 and H1299 cells. Relative enrichment (means \pm SD) represents IncRNA LCETRL3 levels precipitated with TDP43 relative to an input control from three independent experiments. (e) There was markedly increased TDP43 expression in NSCLC tissues compared to normal tissues of the combined samples in the Discovery and Validation cohorts $(n=64)$. (f) The TDP43 protein levels in PC9 and H1299 cells after either silencing of LCETRL3 or over-expressing LCETRL3 without any treatments. $(\mathbf{g}, \mathbf{h})$ Western blot analyses of TDP43 protein levels in PC9 and H1299 cells with stable over-expressed or silenced LCETRL3 after treatment of cycloheximide (CHX). (i) Western blot analyses of the ubiquitination of TDP43 in PC9 and H1299 cells that stabilized either silenced LCETRL3 or over-expressed LCETRL3. (j, k) LCETRL3 obviously upregulated NOTCH1 expression, suppressed PTEN expression and promoted phosphorylation of AKT in PC9 cells (j) and H1299 cells (k). Twotailed unpaired $t$ test, ns, not significant, ${ }^{*} P<0.05,{ }^{* *} P<0.01,{ }^{* * *} P<0.001$ 
or the cytoplasm fraction of NSCLC cells (Fig. 5a). After performing RNA pulldown assays plus mass spectrometry proteomics, we identified 110 proteins (PC9 cells) or 25 proteins (H1299 cells) pulled down by IncRNA LCETRL4 (Fig. 5b). Among four LCETRL4pulled down proteins recognized in both cell lines, EIF2S1 is the $1^{\text {st }}$ most abundant protein (Fig. $5 \mathrm{~b}$ and Supplementary Table 3).
Consistently, we successfully verified EIF2S1 through Western blot using samples of independent RNA pulldown assays in PC9 or H1299 cells (Fig. 5c). RIP assays indicated that IncRNA LCETRL4 could be markedly enriched in the RNA-protein complexes precipitated with the EIF2S1 antibody as compared with the IgG control in NSCLC cells $(P<0.001)$ (Fig. $5 d)$.

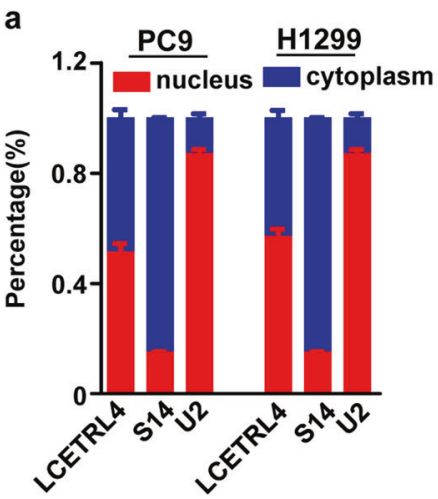

b Proteins identified via
LCETRL4 RNA pulldown

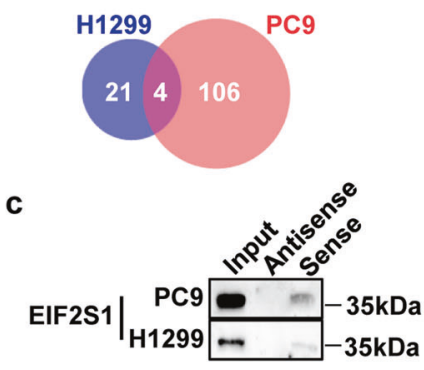

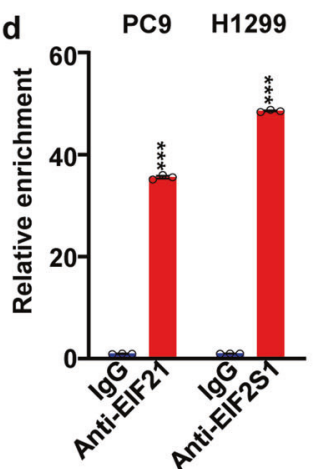

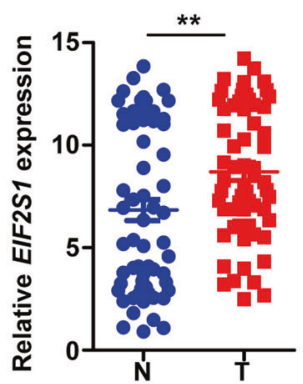

h

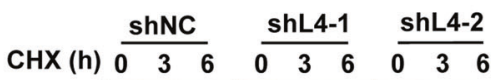

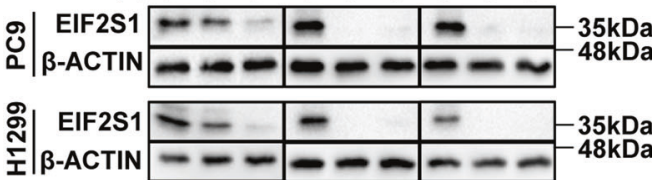

$\mathbf{f}$

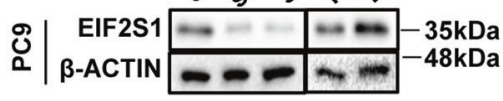

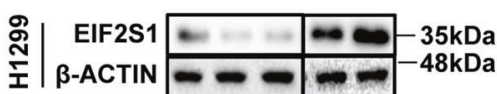

g

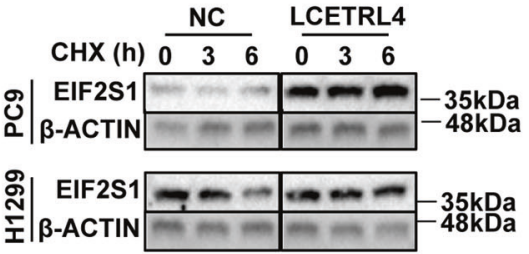

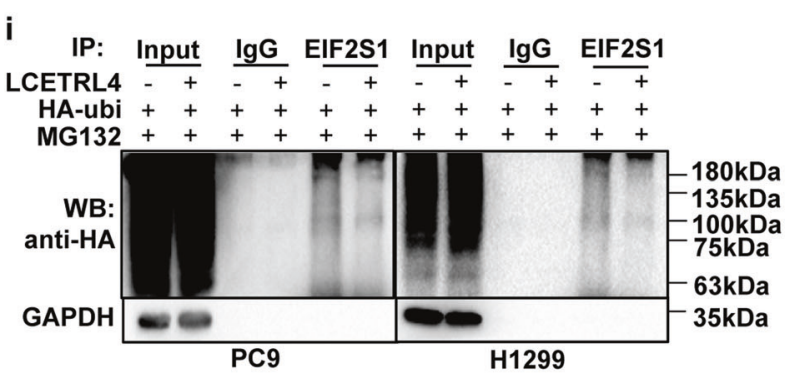

j

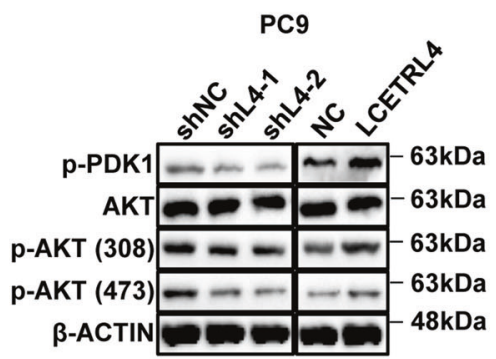

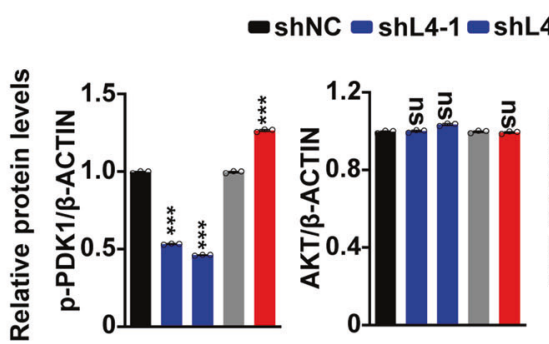

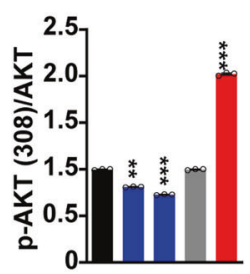

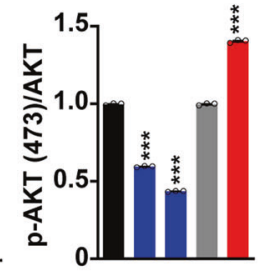

k

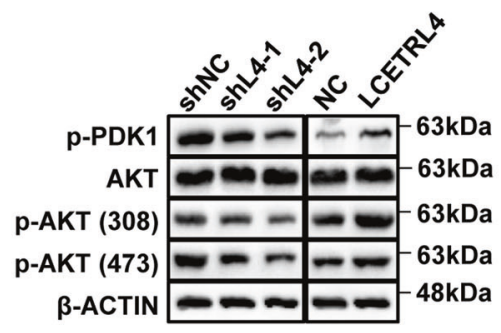

H1299

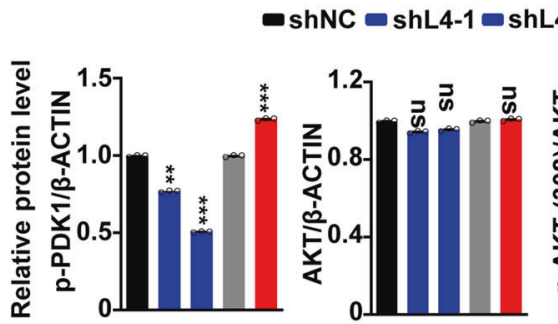

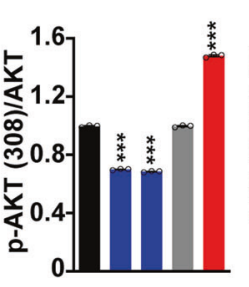


Fig. 5 LncRNA LCETRL4 stabilized EIF2S1 protein and activated the AKT signaling. (a) Cellular location of LCETRL4 in PC9 and H1299 cells. (b) RNA pulldown assays plus mass spectrometry proteomics indicated that there were four proteins pulled down by IncRNA LCETRL4 in PC9 or H1299 cells. (c) Western blot validation of EIF2S1 pulled down by IncRNA LCETRL4. (d) LCETRL4 could be precipitated with antibody against EIF2S1 as compared with the IgG control in PC9 and H1299 cells. Relative enrichment (means \pm SD) represents IncRNA LCETRL4 levels precipitated with EIF2S1 relative to an input control from three independent experiments. (e) There was evidently evaluated EIF2S1 expression in NSCLC tissues compared to normal tissues of the combined samples in Discovery and Validation cohorts $(n=64)$. (f) The EIF2S1 protein levels in PC9 and H1299 cells after either silencing of LCETRL4 or over-expressing LCETRL4 without any treatments. (g, h) Western blot analyses of EIF2S1 protein levels in PC9 and H1299 cells with stable over-expressed or silenced LCETRL4 after treatment of CHX. (i) Western blot analyses of the ubiquitination of EIF2S1 in PC9 and H1299 cells that stabilized either silenced LCETRL4 or over-expressed LCETRL4. (j, k) LCETRL4 significantly enhanced phosphorylation of PDK and AKT in PC9 cells (j) and H1299 cells (k). Two-tailed unpaired $t$ test, ns, not significant, ${ }^{* * *} P<0.01,{ }^{* * * *} P<0.001$

There was remarkably increased EIF2S1 expression in cancerous tissues compared with that in normal tissues in combined samples from the Discovery cohort and Validation cohort $(P<0.01)$ (Fig. 5e). Interestingly, silencing of LCETRL4 evidently suppressed EIF2S1 protein expression in NSCLC cells (Fig. 5f), indicating that LCETRL4 might be involved in the regulation of the proteasome degradation of EIF2S1. Rescue assays indicated that over-expression of EIF2S1 could promote cell growth of the LCETRL4-KD NSCLC cells (Supplementary Fig. 7b). To further confirm whether IncRNA LCETRL4 impacts EIF2S1 degradation, we examined EIF2S1 expression in PC9 and H1299 cells treated with CHX. In LCETRL4-OE PC9 or H1299 cells, LCETRL4 diminished down-regulation of EIF2S1 protein after CHX treatment (Fig. $5 \mathrm{~g}$ ). Conversely, we observed that the EIF2S1 protein levels decreased much faster in the LCETRL4-KD cells compared to the control cells (Fig. 5h). To test if LCETRL4-dependent degradation of EIF2S1 was mediated by its ubiquitination, we examined ubiquitination levels of endogenous EIF2S1 immunoprecipitated in PC9 or H1299 cells transfected with HA-ubi. Obviously decreased ubiquitin signals of EIF2S1 protein were detected in the LCETRL4-OE cells compared to the control cells (Fig. 5i). Taken together, these data revealed that that IncRNA LCETRL4 could stabilize EIF2S1 via repressing its ubiquitination and degradation through proteasome.

EIF2S1 is a subunit of the translation initiation factor EIF2 complex. $^{30,31}$ Interestingly, mouse embryonic fibroblasts with the Eif2s1 mutation grew 50\% slower and showed reduced Pdk1-AktmTOR signaling compared to wild-type cells. ${ }^{31}$ We hypothesized that high levels of LCETRL4 may stabilize EIF2S1, reinforce phosphorylation of PDK1 and, thus, promote activation of the AKT signaling in NSCLC. To test it, we detected levels of these proteins and the phosphorylated ones in PC9 and H1299 cells (Fig. 5j, k). Silencing of LCETRL4 evidently reduced phosphorylation levels of PDK1 (S241) and AKT (T450 and S473); whereas ectopic LCETRL4 improved levels of phosphorylated PDK1 (S241) and phosphorylated AKT (T450 and S473) in cells (Fig. 5j, k). However, dysregulated LCETRL4 did not influence total AKT protein expression in cells (Fig. $5 \mathrm{j}, \mathrm{k}$ ). These results demonstrated that high levels of LCETRL4 could stabilize EIF2S1 and activate the PDK1-AKT signaling in NSCLC.

\section{DISCUSSION}

The chromosome $4 q 12$ is the first GWAS-identified locus associated with PFS of advanced NSCLC patients treated with EGFR-TKIs. ${ }^{7}$ For protein-coding genes at 4q12, NMU which encodes a GPCR ligand was known to be involved in NSCLC progression. $^{32,33}$ However, the biological significance of the noncoding transcripts at $4 q 12$ remains elusive in NSCLC. In the current study, we identified two $4 q 12$ IncRNAs LCETRL3 and LCETRL4 which could dimmish the efficiency of EGFR-TKIs treatments. In line with their oncogenic nature, evidently higher LCETRL3 and LCETRL4 levels were observed in NSCLC tissues as compared with normal specimens. Importantly, IncRNA LCETRL3 can interact with oncoprotein TDP43 and inhibit ubiquitination and degradation of TDP43. Similarly, IncRNA LCETRL4 can bind and stabilize oncoprotein EIF2S1 through reducing ubiquitin- proteasome degradation of EIF2S1. In particular, elevated levels of LCETRL3 or LCETRL4 in NSCLC resulted in high expression of TDP43 or EIF2S1, increased levels of NOTCH1 or phosphorylated PDK1, and, thus, the activated AKT signaling (Fig. 6). Collectively, our data present new clues for interpretation of the genetic differences of individuals receiving EGFR-TKIs therapy from a new perspective and highlight the importance of protein ubiquitination modulated by IncRNAs in NSCLC. ${ }^{34}$

Multiple IncRNAs have been shown to be implicated in malignant development and EGFR-TKIs resistance in NSCLC. ${ }^{16-21}$ For example, tumor suppressor IncRNA GAS5 might play a role in gefitinib resistance which could be reversed by overexpressing GAS5. ${ }^{16}$ Similarly, downregulated expression of IncRNA H19 or HOTAIR contributed to resistance to EGFR-TKls and low levels of H19 or HOTAIR were significantly correlated with shorter PFS in NSCLC patients treated with EGFR-TKIs. ${ }^{18,20}$ On the contrary, several oncogenic IncRNAs (SNHG15, LOC554202 and CRNDE) could promote EGFR-TKIs resistance through different mechanisms. Huang et al found that IncRNA SNHG15 can act as a sponge of miR-451 and facilitate expression of MDR-1 which posing proproliferation, promigration, and antiapoptosis effects on gefitinib-resistant NSCLC cells. ${ }^{19}$ LncRNA LOC554202 upregulated miR-31 expression, repressed RASA1 and FIH-1 expression, and thus, at least partially activated the RAF-MEK-ERK and PI3K-AKT signaling pathways in NSCLC with acquired resistance to gefitinib. $^{17}$ Inhibition of IncRNA CRNDE activated the elF4A3/ MUC1/EGFR signaling and apoptotic activities, and restored sensitivity of NSCLC cells to EGFR-TKIs. ${ }^{21}$ In line with these reports, we for the first time found that IncRNAs LCETRL3 and LCETRL4 at the $4 q 12$ locus are novel oncogenes and reduce the antiproliferation effects of gefitinib on NSCLC cells.

We revealed that IncRNA LCETRL3 can interact with TDP43 protein and suppress degradation of TDP43, an RNA-binding protein that participates in multiple steps of RNA metabolism, including transcription, splicing, and transport of mRNA, as well as miRNA metabolism. ${ }^{24}$ TDP43 has been shown to promote growth and metastasis of NSCLC, triple-negative breast cancer, neuroblastoma, hepatocellular carcinoma and melanoma. ${ }^{35-40}$ Consistently, we found that TDP43 was significantly upregulated in NSCLC tissues. It has been reported that Lys-48 and Lys-63 are two major ubiquitination sites of TDP43 and both sites are linked with polyubiquitin chains. ${ }^{41,42}$ However, TDP-43 of the Lys-48-linked polyubiquitin chains undergoes ubiquitin proteasomal-mediated degradation, while the TDP-43 with Lys-63-linked polyubiquitin chains might be removed via autophagy. ${ }^{43}$ In NSCLC, InCRNA LCETRL3 may mainly block ubiquitination of Lys-48 through binding to TDP43, inhibit proteasomal-mediated degradation of TDP43 and, thus, stabilize oncoprotein TDP43. Our study also demonstrated that increased expression of TDP43 controlled by IncRNA LCETRL3 could stabilize the NOTCH1 mRNA, elevate NOTCH1 expression, downregulate PTEN expression and activate AKT in NSCLC cells, which thereby accelerating tumor growth and reduce EGFR-TKIs efficiency.

In eukaryotic cells, the aberrant accumulation of unfolded or misfolded proteins at the endoplasmic reticulum (ER) induces ER stress, which is emerging as a possible driver of human cancers 

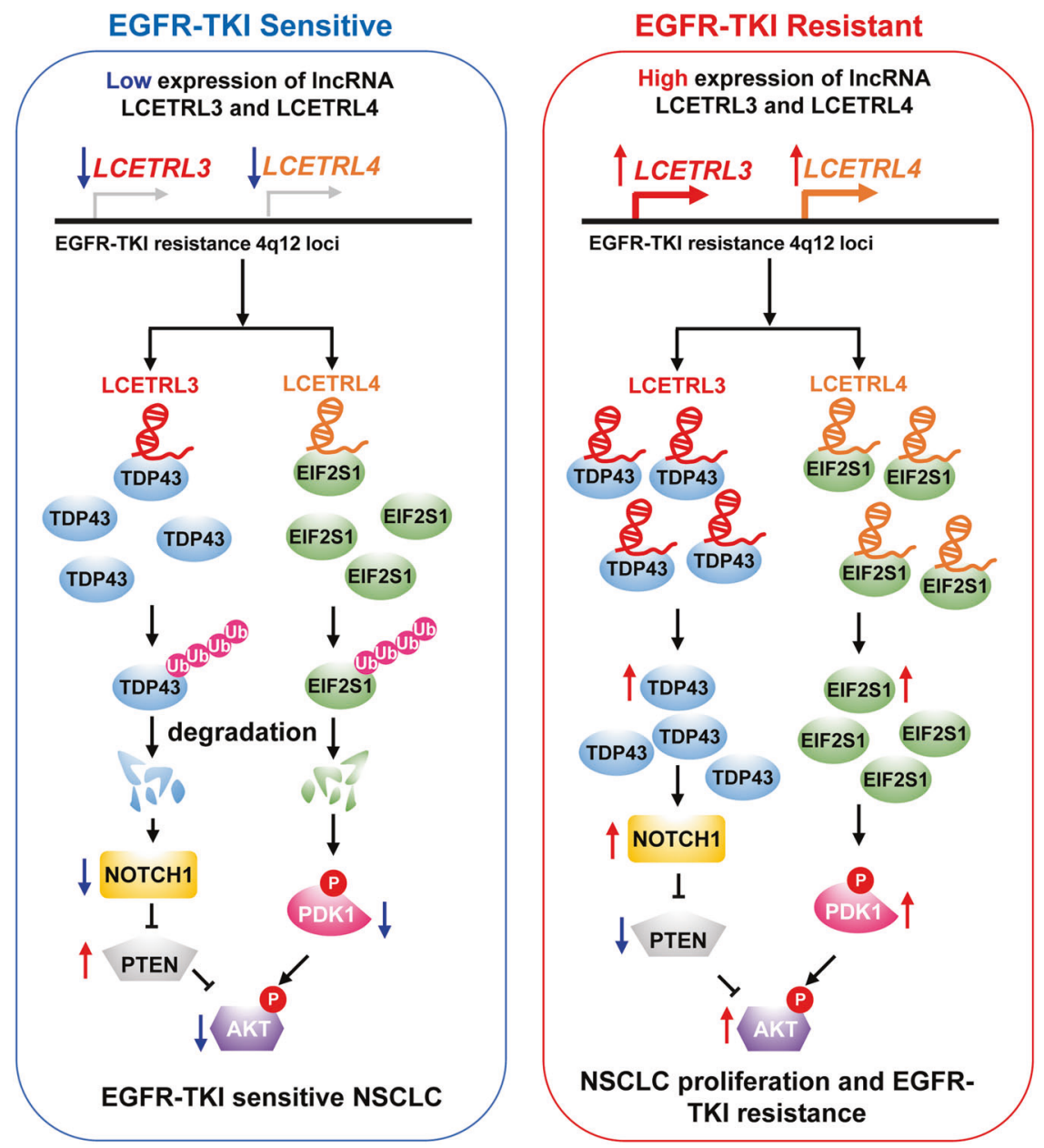

Fig. 6 Graphical representation of the oncogenic functions of IncRNA LCETRL3 or LCETRL4 in EGFR-TKIs treatment of NSCLC. In EGFR-TKIs sensitive cells, low levels of IncRNA LCETRL3 or LCETRL4 cause deactivation of the AKT signaling in NSCLC. However, high levels of LCETRL3 or LCETRL4 could stabilize and upregulate oncoproteins TDP43 or EIF2S1, increase levels of NOTCH1 or phosphorylated PDK1, activate the AKT signaling and, thus, result in EGFR-TKIs resistance of NSCLC cells

and contributes to resistance to chemotherapy, target therapy and immunotherapy. ${ }^{44-46}$ EIF2S1, also known as EIF2a, plays a key role in the PERK-EIF2a signaling, which is one of three major ER stress branches. PERK phosphorylates elF2a and reduces the overall frequency of mRNA translation initiation under ER stress. ${ }^{44-46}$ Of note, mouse embryonic fibroblasts carrying the Eif2s1 mutation showed reduced activation of the Pdk1-Akt signaling. ${ }^{31}$ In line with this, we observed interactions between LCETRL4 and EIF2S1 lead to elevated EIF2S1 expression, deceased levels of phosphorylated PDK1 and stimulated AKT signaling.

In summary, we revealed a novel model that integrates two IncRNAs transcribed from the $4 q 12$ locus into the regulation of oncogenic programs and EGFR-TKIs resistance in NSCLC. These findings shed new light on the importance of functionally annotating IncRNAs in the GWAS loci and provided insights to declare EGFR-TKIs resistance mechanisms developed by cancer cells. Considering that rapid development of resistance to targeted therapy drugs represents a major challenge for managing NSCLC, we believe that identification of novel druggable targets, i.e., IncRNAs, may unlock the therapeutic potential of NSCLC in the clinic.

\section{MATERIAL AND METHODS}

Patients and tissue specimens

There are two NSCLC patient cohorts (Discovery cohort and Validation cohort) in this study. In the Discovery cohort, twenty
NSCLC patients were recruited at Shandong Cancer Hospital and Institute (Jinan, Shandong Province, China) between October 2016 and June 2017. In the Validation cohort, forty-four NSCLC patients were recruited at Shandong Cancer Hospital and Institute between July 2017 and November 2019. All stage I or II NSCLC patients received curative surgical resection. For the stage, Illa NSCLC patients, surgical resection of the primary tumor site was performed to alleviate symptoms if patients were tolerable for the surgery. Chemotherapy and/or radiotherapy were given to these stage IIla NSCLC patients after surgery. Fresh cancerous specimens and normal lung tissues were sampled from these patients. Normal lung tissues were obtained at least $2 \mathrm{~cm}$ away from the NSCLC border. All cases were Han Chinese. The detailed characteristics of all patients were shown in Supplementary Table 4. This study was approved by the institutional review board of Shandong Cancer Hospital and Institute. At recruitment, written informed consent was obtained from each subject. The methods were carried out in accordance with the approved guidelines.

Quantitative reverse transcription PCR (RT-qPCR)

Total RNA from cultured cells or tissue specimens was isolated with Trizol reagent (Invitrogen, 94402). Each RNA sample was treated with DNase I (RNase-free) to remove genomic DNA (Thermo Fisher, 18068015). Each RNA sample was then reversetranscribed into cDNAs using PrimeScript ${ }^{\mathrm{TM}}$ RT Master Mix 
10

(TaKaRa, RR036A). Relative RNA levels of candidate genes were calculated by using the $2^{-\Delta \Delta \mathrm{Ct}}$ method. Indicated primers are listed in Supplementary Table 5 . Each sample was examined at least in triplicate. PCR product specificity was confirmed by melting-curve analyses.

\section{Cell culture}

Human NSCLC H1299 cell line was obtained from the Shanghai Cell Collection, Chinese Academy of Sciences. Human NSCLC PC9 cells was purchased from JENNIO Biological Technology. HEK293T cells were kindly provided by Dr. Yunshan Wang (Jinan Central Hospital, Shandong Province, China). Cells were cultured in Dulbecco's modified Eagle's medium (DMEM) (Gibco, C11995500BT) with $10 \%$ fetal bovine serum (FBS; Gibco, 1347575). Cells were maintained at $37^{\circ} \mathrm{C}$ in a $5 \% \mathrm{CO}_{2}$ incubator and periodically tested and found to be negative for mycoplasma.

RNA interference assays

Small interference RNAs (siRNAs) of LCETRL3 (siL3-1 and siL3-2), LCETRL4 (siL4-1 and siL4-2) as well as the negative control RNA (NC RNA) were ordered from Genepharma (Shanghai, China) (Supplementary Table 6). As reported previously, ${ }^{10,12}$ all small RNAs were transfected to PC9 and H1299 cells using the INTERFERin reagent (Polyplus, 409-10).

The expression and shRNA constructs of LCETRL3 or LCETRL4 The full-length IncRNA LCETRL3 or LCETRL4 CDNA was directly synthesized by Genewiz (Suzhou, Jiangsu Province, China) and cloned after the CMV promoter of the PCDH-CMV-MCS-EF1-Puro vector. The plasmid was named as LCETRL3 or LCETRL4. Two shRNA hairpins targeting human LCETRL3 (shLCETRL3-1 or shLCETRL3-2) or LCETRL4 (shLCETRL4-1 or shLCETRL4-2) or the control shRNA (Supplementary Table 7) were cloned into the pLKO.1 vector. The resultant plasmids were designated ShLCETRL3-1, shLCETRL3-2, shLCETRL4-1, shLCETRL4-2, or shNC. All aforementioned plasmids were sequenced to confirm the orientation and integrity.

\section{Lentiviral transduction}

The lentivirus LCETRL3, LCETRL4, shLCETRL3-1, shLCETRL3-2, shLCETRL4-1, shLCETRL4-2 plasmid was co-transfected into HEK293T cells with the psPAX2 (Addgene, \#12260) and pMD2.G (Addgene, \#12259) plasmids using the jetPRIME reagent (Polyplus, 114-07) as reported previously. ${ }^{23,47}$ At $48 \mathrm{~h}$ and $72 \mathrm{~h}$ following transfection, recombinant lentiviral particles of LCETRL3, LCETRL4, shLCETRL3-1, shLCETRL3-2, shLCETRL4-1, shLCETRL4-2 and their controls (NC or shNC) in the viral supernatants were collected. Human PC9 and H1299 cells were infected with the LCETRL3, LCETRL4， shLCETRL3-1， shLCETRL3-2， shLCETRL4-1， shLCETRL4-2, NC or shNC viral supernatants. NSCLC cells were then selected using $2 \mu \mathrm{g} / \mathrm{mL}$ puromycin. In these lentiviral transducted cells, the expression levels of LCETRL3 or LCETRL4 were examined by RT-qPCR.

Cell proliferation and gefitinib drug sensitivity analyses

For cell proliferation assays, a total of $5 \times 10^{4}$ of PC9 cells or $3 \times 10^{4}$ H1299 cells after lentiviral transduction of LCETRL3-knockdown (KD), LCETRL4-KD, LCETRL3-overexpression (OE) or LCETRL4-OE were seeded in 12-well plates. NSCLC cells were harvested and counted at 24,48 , and $72 \mathrm{~h}$ after seeding. For gefitinib drug sensitivity analyses, a total of 7500 cells of the stably transfected PC9 and H1299 cells were seeded per well in 96-well plates. Gefitinib diluted in DMSO was added to each well to achieve the desired final concentrations (PC9: $0 \mu \mathrm{mol} / \mathrm{L}, 2.5 \mu \mathrm{mol} / \mathrm{L}, 5 \mu \mathrm{mol} / \mathrm{L}$, $7.5 \mu \mathrm{mol} / \mathrm{L}$, and $10 \mu \mathrm{mol} / \mathrm{L} ; \mathrm{H} 1299: 0 \mu \mathrm{mol} / \mathrm{L}, 20 \mu \mathrm{mol} / \mathrm{L}, 40 \mu \mathrm{mol} / \mathrm{L}$, $60 \mu \mathrm{mol} / \mathrm{L}$, and $80 \mu \mathrm{mol} / \mathrm{L})$ as described previously. ${ }^{9}$ After cells were incubated with gefitinib for $48 \mathrm{~h}, 10 \mu \mathrm{L}$ of $0.5 \mathrm{mg} / \mathrm{mL} \mathrm{MTT}$ labeling reagents (Sigma, \#88417, Temecula, CA, USA) were added to each well. One hundred microliters of DMSO were added to each well after the cells were incubated with MTT for $4 \mathrm{~h}$ in a cell culture incubator. Absorbance in each well was measured at $570 \mathrm{~nm}$ using a microplate reader.

Colony formation assays

The stably LCETRL3-KD, LCETRL4-KD, LCETRL3-OE, or LCETRL4-OE PC9 $(2,000$ cells per well) were seeded into a 6 -well cell culture plate. When colonies were visible after 8 days, cells were washed with cold PBS twice and fixed with the fixation fluid (methanol: acetic acid = 3:1). The cells were dyed with crystal violet and the number of the NSCLC colony in each well was counted. A total of 500 stably LCETRL3-KD and LCETRL4-KD H1299 cells or 2000 stably LCETRL3-OE and LCETRL4-OE H1299 cells per well were seeded into a 6-well cell culture plate. For the LCETRL3-KD and LCETRL4-KD H1299 cells, the NSCLC colony number was counted after 16 days. The colony number of the LCETRL3-OE and LCETRL4-OE H1299 cells was counted after 8 days.

NSCLC xenografts

To evaluate the in vivo role of LCETRL3 and LCETRL4, we inoculated subcutaneously a total of $6 \times 10^{6}$ LCETRL3-OE, LCETRL4-OE or control (NC) PC9 cells into fossa axillaries of fiveweek-old female nude BALB/c mice (Vital River Laboratory, Beijing, China) ( $n=5$ per group). Tumor growth was measured every two days after tumor volumes equaled to $100 \mathrm{~mm}^{3}$. To examine the involvement of LCETRL3 and LCETRL4 in gefitinib drug sensitivity in vivo, similar LCETRL3-OE, LCETRL4-OE or NC PC9 xenografts $(n=$ 5 per group) were obtained as described previously. ${ }^{23,47}$ At the 8 th day (tumor volumes equaled to or exceeded $200 \mathrm{~mm}^{3}$ ), $15 \mathrm{mg} / \mathrm{kg}$ gefitinib per mouse was given daily by intragastric administration. Frozen section and $\mathrm{H} \& \mathrm{E}$ staining were processed as described previously. ${ }^{23,47}$ All procedures involving mice were approved by the institutional Animal Care Committee of Shandong Cancer Hospital and Institute.

\section{Transwell assays}

The transwell chambers (pore $8 \mathrm{~mm}$, Corning) were firstly coated with $60 \mu \mathrm{L}$ BD Biosciences Matrigel (1:20 dilution) for $12 \mathrm{~h}$ in a $5 \%$ $\mathrm{CO}_{2}$ incubator. PC9 or $\mathrm{H} 1299$ cells were added to upper transwell chambers, with a total of $650 \mu \mathrm{L}$ medium with $10 \%$ FBS in the lower wells. After $48 \mathrm{~h}$, cells migrated to the lower wells through pores were stained with $0.2 \%$ crystal violet solution and counted.

Subcellular fractionation

The cytosolic and nuclear fractions of PC9 or H1299 cells were separately isolated using the nuclear/cytoplasmic Isolation Kit (Biovision, \#P0027, China) according to the manufacturer's instructions. The relative levels of LCETRL3 or LCETRL4 in cytosolic or nuclear fractions were detected by qRT-PCR.

\section{RNA pulldown}

RNA pulldown was performed following the standard protocol as reported previously. ${ }^{23,48-50}$ To prepare the plasmid construct template for in vitro RNA synthesis, LCETRL3 or LCETRL4 was subcloned into pcDNA3.1 with inserted T7 promoter before and after the cloning site. After these constructs were linearized, IncRNAs were transcribed with T7 RNA polymerase (MEGAscript T7 Transcript Kit, Thermo fisher, AM1330) and purified with the RNeasy minikit (Qiagen, \#74104, Germany). Pierce ${ }^{\mathrm{TM}}$ RNA 3' End Desthiobiotinylation Kit (Thermo fisher, 20163) was used to biotinylate sense and antisense LCETRL3 or LCETRL4 RNAs. These RNAs were then incubated with PC9 or $\mathrm{H} 1299$ protein extracts at $4{ }^{\circ} \mathrm{C}$ for $1 \mathrm{~h}$. Proteins bound on the streptavidin magnetic beads were eluted with the Elution Buffer of Pierce ${ }^{\text {TM }}$ Magnetic RNAProtein Pull-Down Kit (Thermo, 20164). The recovered proteins were then analyzed by liquid chromatography-tandem mass spectrometry (LS-MS/MS) (Hoogen Biotech Co., Shanghai, China) 
and Western Blot. MaxQuant software (version 1.5.3.30) with the UniProtKB human database (uniport Homo sapiens $188441_{-}$ 20200326) was utilized to analyze mass spectra.

RNA immunoprecipitation (RNA-IP)

Magna RIP RNA-Binding Protein Immunoprecipitation Kit (Millipore, 17-700) with the EIF2S1 and TDP43 antibodies or IgG Isotype-control were used for RNA-IP assays as reported previously. ${ }^{23,47}$ The EIF2S1-RNA or TDP43-RNA complexes were recovered by Dynabeads ${ }^{\circledR}$ Protein $G$ beads (Invitrogen, Lot\#00929981). LnCRNA LCETRL3 or LCETRL4 levels in the precipitates were measured by RT-qPCR.

\section{Western blot}

Western blot was performed as described previously. ${ }^{23,51}$ After extracted from culture cells, total proteins of NSCLC cells were separated with the SDS-PAGE gel and transferred to a polyvinylidene fluoride (PVDF) membrane (Millipore, ISEQ00010). The PVDF membrane was then incubated with antibodies against TDP43, NOTCH1, EIF2S1, AKT, p-AKT (308), p-AKT (473), PTEN, p-PDK1, GAPDH or $\beta$-ACTIN (Supplementary Table 8 ) overnight at $4{ }^{\circ} \mathrm{C}$. Proteins were visualized with ECL Western Blotting Substrate (Pierce, 32106).

\section{Turnover assays}

The stably LCETRL3-KD, LCETRL4-KD, LCETRL3-OE or LCETRL4-OE PC9 and $\mathrm{H} 1299$ were seeded into 6-well plates and then cultured for $24 \mathrm{~h}$. To stop de novo protein synthesis, cells were incubated with $\mathrm{CHX}$ (\# 66-81-9, Merck, US) at a final concentration of $200 \mu \mathrm{g} / \mathrm{mL}$. At the indicated times after CHX treatments, the PC9 and H1299 cells were harvested. Western blot was performed to examine the TDP43, EIF2S1 and $\beta$-ACTIN protein levels in NSCLC cells.

\section{Ubiquitination assays}

Ubiquitination assays were carried out in PC9 and H1299 cells which were transfected with the pcDNA3.1-HA-ubiquitin (HA-ubi) plasmid as reported previously. ${ }^{23}$ At $36 \mathrm{~h}$ after transfection, NSCLC cells were incubated with $50 \mu \mathrm{g} / \mathrm{mL}$ MG132 for $6 \mathrm{~h}$ and then lysed with the ice-cold RIPA buffer (Beyotime, P0013C). To isolate ubiquitinated TDP43 or EIF2S1, proteins in the cell lysate were immunoprecipitated with anti-TDP43 or anti-EIF2S1 antibodies and then detected with the anti-HA antibody by Western blot.

\section{Statistics}

The 2-tailed unpaired Student's $t$ test or paired Student's $t$ test was used to examine differences between two groups. The criterion of statistical significance was a $P$ value of less than 0.05 . All analyses were performed with GraphPad Prism (Version 5.0, GraphPad Software, Inc.).

\section{DATA AVAILABILITY}

The additional data collected during this study are available from the corresponding author upon reasonable request.

\section{ACKNOWLEDGEMENTS}

This work was supported by Taishan Scholars Program of Shandong Province (tsqn20161060) (to M.Y.); National Natural Science Foundation of China (82173070 and 31871306) (to M.Y.); Natural Science Foundation of Shandong Province (ZR2021LZL004) (to M.Y.); Program of Science and Technology for the youth innovation team in universities of Shandong Province (2020KJL001) (to M.Y.).

\section{AUTHOR CONTRIBUTIONS}

M.Y. conceived and designed this study. Y.L. performed the experiments. M.Y. and Y. L. acquired, analyzed, and interpreted the data from experiments. Y.L., Y.S., M.X., J.Z., B.W., and T.W. collected the human samples. M.Y. and Y.L. drafted the manuscript. M.Y. and J.Y. critically revised the manuscript for important intellectual content. M.Y. and J.Y. supervised this study. All authors have read and approved the article.

\section{ADDITIONAL INFORMATION}

Supplementary information The online version contains supplementary material available at https://doi.org/10.1038/s41392-021-00847-2.

Competing interests: The authors declare no competing interests.

\section{REFERENCES}

1. Sung, $H$. et al. Global cancer statistics 2020: GLOBOCAN estimates of incidence and mortality worldwide for 36 cancers in 185 countries. CA Cancer J. Clin. 71, 209-249 (2021)

2. Zhou, C. et al. Final overall survival results from a randomized, phase III study of erlotinib versus chemotherapy as first-line treatment of EGFR mutation-positive advanced non-small-cell lung cancer (OPTIMAL, CTONG-0802). Ann. Oncol. 26, 1877-1883 (2015).

3. Wu, Y. L. et al. Efficacy according to blind independent central review: Post-hoc analyses from the phase III, randomized, multicenter, IPASS study of first-line gefitinib versus carboplatin/paclitaxel in Asian patients with EGFR mutationpositive advanced NSCLC. Lung cancer 104, 119-125 (2017).

4. Noronha, V. et al. Gefitinib Versus Gefitinib Plus Pemetrexed and Carboplatin Chemotherapy in EGFR-Mutated Lung Cancer. J. Clin. Oncol. 38, 124-136 (2020).

5. Hosomi, Y. et al. Gefitinib Alone Versus Gefitinib Plus Chemotherapy for NonSmall-Cell Lung Cancer With Mutated Epidermal Growth Factor Receptor: NEJ009 Study. J. Clin. Oncol. 38, 115-123 (2020).

6. Arteaga, C. L. \& Engelman, J. A. ERBB receptors: from oncogene discovery to basic science to mechanism-based cancer therapeutics. Cancer Cell 25, 282-303 (2014).

7. Chang, I. S. et al. Genetic Modifiers of Progression-Free Survival in Never-Smoking Lung Adenocarcinoma Patients Treated with First-Line Tyrosine Kinase Inhibitors. Am. J. Respir. Crit. Care. Med 195, 663-673 (2017).

8. $\mathrm{Ng}, \mathrm{K}$. P. et al. A common BIM deletion polymorphism mediates intrinsic resistance and inferior responses to tyrosine kinase inhibitors in cancer. Nat. Med 18, 521-528 (2012).

9. Li, J. et al. Leukocyte Telomere Length and Clinical Outcomes of Advanced Lung Adenocarcinoma Patients with Epidermal Growth Factor Receptor Tyrosine Kinase Inhibitors Treatment. DNA Cell Biol. 37, 903-908 (2018).

10. Zhang, N. et al. miR- 608 and miR-4513 significantly contribute to the prognosis of lung adenocarcinoma treated with EGFR-TKIs. Lab. Invest 99, 568-576 (2019).

11. Zhang, N. et al. DACT2 modulated by TFAP2A-mediated allelic transcription promotes EGFR-TKIs efficiency in advanced lung adenocarcinoma. Biochem. Pharm. 172, 113772 (2020)

12. Li, Y. et al. Oncogene HSPH1 modulated by the rs2280059 genetic variant diminishes EGFR-TKIs efficiency in advanced lung adenocarcinoma. Carcinogenesis 41, 1195-1202 (2020).

13. Huarte, M. The emerging role of IncRNAs in cancer. Nat. Med 21, 1253-1261 (2015).

14. Evans, J. R., Feng, F. Y. \& Chinnaiyan, A. M. The bright side of dark matter: IncRNAs in cancer. J. Clin. Invest 126, 2775-2782 (2016).

15. Anastasiadou, E., Jacob, L. S. \& Slack, F. J. Non-coding RNA networks in cancer. Nat. Rev. Cancer 18, 5-18 (2018).

16. Dong, S. et al. The long non-coding RNA, GAS5, enhances gefitinib-induced cell death in innate EGFR tyrosine kinase inhibitor-resistant lung adenocarcinoma cells with wide-type EGFR via downregulation of the IGF-1R expression. J. Hematol. Oncol. 8, 43 (2015).

17. He, J. et al. Long non-coding RNA LOC554202 promotes acquired gefitinib resistance in non-small cell lung cancer through upregulating miR-31 expression. J. Cancer 10, 6003-6013 (2019).

18. Chen, $\mathrm{C}$. et al. LncRNA H19 downregulation confers erlotinib resistance through upregulation of PKM2 and phosphorylation of AKT in EGFR-mutant lung cancers. Cancer Lett. 486, 58-70 (2020).

19. Huang, J. et al. LncRNA SNHG15 regulates EGFR-TKI acquired resistance in lung adenocarcinoma through sponging miR-451 to upregulate MDR-1. Cell Death Dis. 11, 525 (2020).

20. Wang, Q. et al. HOTAIR induces EGFR-TKIs resistance in non-small cell lung cancer through epithelial-mesenchymal transition. Lung cancer 147, 99-105 (2020)

21. Takahashi, S. et al. Long Non-Coding RNA CRNDE Is Involved in Resistance to EGFR Tyrosine Kinase Inhibitor in EGFR-Mutant Lung Cancer via elF4A3/MUC1/ EGFR Signaling. Int. J. Mol. Sci. 22, 4005 (2021).

22. Pan, W. et al. The long noncoding RNA GAS8-AS1 suppresses hepatocarcinogenesis by epigenetically activating the tumor suppressor GAS8. J. Biol. Chem. 293, 17154-17165 (2018)

23. Yuan, J. et al. LncRNA SLC26A4-AS1 suppresses the MRN complex-mediated DNA repair signaling and thyroid cancer metastasis by destabilizing DDX5. Oncogene 39, 6664-6676 (2020).

24. Dunker, W. et al. TDP-43 prevents endogenous RNAs from triggering a lethal RIGI-dependent interferon response. Cell Rep. 35, 108976 (2021). 
25. Modic, M. et al. Cross-Regulation between TDP-43 and Paraspeckles Promotes Pluripotency-Differentiation Transition. Mol. Cell 74, 951-965 e913 (2019).

26. Nishino, K. et al. Mice deficient in the C-terminal domain of TAR DNA-binding protein 43 develop age-dependent motor dysfunction associated with impaired Notch1-Akt signaling pathway. Acta Neuropathol. Commun. 7, 118 (2019).

27. Palomero, T. et al. Mutational loss of PTEN induces resistance to NOTCH1 inhibition in T-cell leukemia. Nat. Med 13, 1203-1210 (2007).

28. Graziani, I. et al. Opposite effects of Notch-1 and Notch-2 on mesothelioma cell survival under hypoxia are exerted through the Akt pathway. Cancer Res 68, 9678-9685 (2008).

29. Eliasz, S. et al. Notch-1 stimulates survival of lung adenocarcinoma cells during hypoxia by activating the IGF-1R pathway. Oncogene 29, 2488-2498 (2010).

30. Scheuner, D. et al. Control of mRNA translation preserves endoplasmic reticulum function in beta cells and maintains glucose homeostasis. Nat. Med 11, 757-764 (2005).

31. Yung, H. W. et al. Endoplasmic reticulum stress disrupts placental morphogenesis: implications for human intrauterine growth restriction. J. Pathol. 228, 554-564 (2012).

32. Takahashi, K. et al. The neuromedin U-growth hormone secretagogue receptor $1 \mathrm{~b} /$ neurotensin receptor 1 oncogenic signaling pathway as a therapeutic target for lung cancer. Cancer Res 66, 9408-9419 (2006).

33. $\mathrm{Wu}, \mathrm{Y}$. et al. Neuromedin $\mathrm{U}$ is regulated by the metastasis suppressor RhoGDI2 and is a novel promoter of tumor formation, lung metastasis and cancer cachexia. Oncogene 26, 765-773 (2007).

34. Zhou, J. et al. Implications of protein ubiquitination modulated by IncRNAs in gastrointestinal cancers. Biochem. Pharm. 188, 114558 (2021).

35. Guo, F. et al. Regulation of MALAT1 expression by TDP43 controls the migration and invasion of non-small cell lung cancer cells in vitro. Biochem. Biophys. Res. Commun. 465, 293-298 (2015).

36. Lin, T. W. et al. TDP-43/HDAC6 axis promoted tumor progression and regulated nutrient deprivation-induced autophagy in glioblastoma. Oncotarget 8, 56612-56625 (2017).

37. Zeng, Q. et al. Identification of TDP- 43 as an oncogene in melanoma and its function during melanoma pathogenesis. Cancer Biol. Ther. 18, 8-15 (2017).

38. Ke, H. et al. Loss of TDP43 inhibits progression of triple-negative breast cancer in coordination with SRSF3. Proc. Natl Acad. Sci. USA 115, E3426-E3435 (2018).

39. Chen, X. et al. TDP-43 regulates cancer-associated microRNAs. Protein Cell 9, 848-866 (2018)

40. Guo, F. et al. TDP-43 induces EMT and promotes hepatocellular carcinoma metastasis via activating Wnt/beta-catenin signaling pathway. Am. J. Cancer Res 10, 3285-3301 (2020).
41. Hebron, M. L. et al. Parkin ubiquitinates Tar-DNA binding protein-43 (TDP-43) and promotes its cytosolic accumulation via interaction with histone deacetylase 6 (HDAC6). J. Biol. Chem. 288, 4103-4115 (2013).

42. Seyfried, N. T. et al. Multiplex SILAC analysis of a cellular TDP-43 proteinopathy model reveals protein inclusions associated with SUMOylation and diverse polyubiquitin chains. Mol. Cell Proteom. 9, 705-718 (2010).

43. Scotter, E. L. et al. Differential roles of the ubiquitin proteasome system and autophagy in the clearance of soluble and aggregated TDP-43 species. J. Cell. Sci. 127, 1263-1278 (2014).

44. King, A. P. \& Wilson, J. J. Endoplasmic reticulum stress: an arising target for metalbased anticancer agents. Chem. Soc. Rev. 49, 8113-8136 (2020).

45. Chen, X. \& Cubillos-Ruiz, J. R. Endoplasmic reticulum stress signals in the tumour and its microenvironment. Nat. Rev. Cancer 21, 71-88 (2021).

46. Hetz, C., Axten, J. M. \& Patterson, J. B. Pharmacological targeting of the unfolded protein response for disease intervention. Nat. Chem. Biol. 15, 764-775 (2019).

47. Tian, B. et al. Oncogenic SNORD12B activates the AKT-mTOR-4EBP1 signaling in esophageal squamous cell carcinoma via nucleus partitioning of PP-1alpha. Oncogene 40, 3734-3747 (2021).

48. Xing, Z. et al. IncRNA directs cooperative epigenetic regulation downstream of chemokine signals. Cell 159, 1110-1125 (2014).

49. Zhang, Y. et al. The IncRNA Snhg1-Vps13D vesicle trafficking system promotes memory CD8 T cell establishment via regulating the dual effects of IL-7 signaling. Signal Transduct. Target Ther. 6, 126 (2021).

50. Xing, Z. et al. Expression of Long Noncoding RNA YIYA Promotes Glycolysis in Breast Cancer. Cancer Res 78, 4524-4532 (2018).

51. Zhang, N. et al. MED13L integrates Mediator-regulated epigenetic control into lung cancer radiosensitivity. Theranostics 10, 9378-9394 (2020).

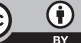

Open Access This article is licensed under a Creative Commons Attribution 4.0 International License, which permits use, sharing, adaptation, distribution and reproduction in any medium or format, as long as you give appropriate credit to the original author(s) and the source, provide a link to the Creative Commons license, and indicate if changes were made. The images or other third party material in this article are included in the article's Creative Commons license, unless indicated otherwise in a credit line to the material. If material is not included in the article's Creative Commons license and your intended use is not permitted by statutory regulation or exceeds the permitted use, you will need to obtain permission directly from the copyright holder. To view a copy of this license, visit http://creativecommons. org/licenses/by/4.0/.

(c) The Author(s) 2021 